\title{
LEVANTAMENTO NUTRICIONAL DOS BANANAIS DA REGIÃO NORTE DE MINAS GERAIS PELA ANÁLISE FOLIAR ${ }^{1}$
}

\author{
ENILSON DE BARROS SILVA², MARIA GERALDA VILELA RODRIGUES ${ }^{3}$
}

\begin{abstract}
RESUMO - O trabalho objetivou avaliar a situação do estado nutricional das bananeiras irrigadas da Região Norte de Minas Gerais, utilizando os resultados das amostras foliares enviadas por produtores, para serem analisadas no Laboratório de Solo e Folha da EPAMIG/CTNM, no ano de 1999, totalizando 1099 amostras. Foram determinados os teores de N, P, K, Ca, Mg, S, B, Cu, Fe, Mn, Zn e $\mathrm{Na}$. Observou-se que $97 \%$ do total das amostras avaliadas apresentaram alguma deficiência, sendo que $36 \%$ para macronutrientes e 95\% para micronutrientes. A ordem decrescente de porcentagem de amostras deficientes em macronutrientes foi $\mathrm{K}=\mathrm{S}>\mathrm{P}>\mathrm{N}>\mathrm{Mg}$ $>\mathrm{Ca}$. Para os micronutrientes, a porcentagem decrescente de amostras que apresentaram deficiência foi $\mathrm{Zn}>\mathrm{Cu}>\mathrm{Fe}>\mathrm{Mn}>\mathrm{B}$. O Ca e Mn foram os que se apresentaram em maior porcentagem das amostras avaliadas com teor adequado de 98 e $87 \%$, respectivamente. O B foi o micronutriente mais freqüentemente encontrado em teor excessivo com $94 \%$ das amostras foliares. Para o Na, o teor foliar entre 10 a $1348 \mathrm{mg} \mathrm{kg}^{-1}$.
\end{abstract}

Termos para indexação: Musa sp., banana, elementos foliares, análise de tecidos.

\section{NUTRICIONAL SURVEY OF THE BANANA CROPS OF THE NORTHERM OF MINAS GERAIS FOR THE FOLIAR ANALYSIS}

\begin{abstract}
The work objectified to evaluate the situation of the state nutricional of the irrigated banana tree of the Northerm of Minas Gerais, using the results of the foliar samples sent by producers, for they be analyzed in the EPAMIG/CTNM Soil and Leaf Laboratory in the year of 1.999 in a total of 1,099 samples. There were analysed the contents of N, P, K, Ca, Mg, S, B, Cu, Fe, Mn, Zn and $\mathrm{Na}$. It was observed $97 \%$ of the appraised samples presented some deficiency, and $36 \%$ of macronutrients and $95 \%$ of micronutrients. The decreasing order of percentage of deficient samples in macronutrients was $\mathrm{K}=\mathrm{S}>\mathrm{P}>\mathrm{N}>\mathrm{Mg}>\mathrm{Ca}$. For the micronutrients the decreasing percentage of samples that presented deficiency was $\mathrm{Zn}>\mathrm{Cu}>\mathrm{Fe}>\mathrm{Mn}>\mathrm{B}$. The $\mathrm{Ca}$ and $\mathrm{Mn}$ were the ones that they appraised in larger percentage of the samples evaluated with appropriate content of 98 and $87 \%$, respectively. The B was the micronutrient more frequenty found in excessive content with $94 \%$ of the foliar samples. For the $\mathrm{Na}$, the foliar content among 10 to $1,348 \mathrm{mg} \mathrm{kg}^{-1}$.
\end{abstract}

Index terms: Musa sp., banana, elements foliar, tissue analysis.

A bananeira é um planta exigente em nutrientes por produzir grande massa vegetativa e ainda apresentar elevadas quantidades de nutrientes absorvidos pela planta e exportados pelos frutos, principalmente nitrogênio $(\mathrm{N})$ e potássio $(\mathrm{K})$ (MartinPrével, 1962; Montagut \& Martin-Prével, 1965; Martin-Prével et al., 1968; Gallo et al., 1972; Borges \& Silva, 1995).

A diagnose foliar é um método de avaliação do estado nutricional das culturas em que se coleta a amostra usando uma determinada folha em época definida durante o desenvolvimento da planta (Malavolta et al., 1997). Como as folhas são utilizadas para análise foliar por serem órgãos de maior atividade química, facilita o diagnóstico de deficiências até toxidez (Malavolta, 1992).

Uma das grandes utilidades da diagnose foliar consiste no levantamento nutricional das lavouras, na propriedade agrícola, de região ou de Estado, trazendo relevante contribuição quando os dados de análise de folhas são acompanhados de produção (Malavolta et al., 1997). O levantamento dos teores foliares tem a finalidade de identificar a existência de deficiências e excessos de nutrientes para direcionar as pesquisas regionais de adubação a serem programadas.

Trabalhos de levantamento do estado nutricional de bananais encontram-se realizados por Gallo et al. (1974) em cultivares de Nanica e Nanicão do Estado de São Paulo. Os autores verificaram que a porcentagem de plantações deficientes eram: $\mathrm{N}=30$ a $40 ; \mathrm{K}=10$ a 20; Ca quase $100 ; \mathrm{Mg}=40$ a 60 e os demais nutrientes não se apresentaram em níveis deficientes.

Malburg et al. (1984), em levantamento nutricional de 22 bananais da cultivar Enxerto, 18 da cultivar Nanicão e 04 da cultivar Branca, observaram, de modo geral, que os níveis foliares estiveram dentro da faixa de normalidade, mesmo em bananais que aparentemente apresentavam sintomas de deficiências.

O trabalho teve por objetivo avaliar a situação do estado nutricional dos bananais do Norte de Minas Gerais, utilizando, para tanto, os resultados das amostras foliares rotineiramente analisadas no Laboratório de Solo da EPAMIG/CTNM, no ano de 1999.

No período de janeiro a dezembro de 1999, foram analisadas 1.099 amostras foliares de bananeira, no Laboratório

1 (Trabalho 189/2000). Recebido: 01/09/2000. Aceito para publicação: 04/10/2001.

2 Eng. Agr., DSc., Pesquisador EPAMIG/CTNM, C.P. 12, 39440-000, Nova Porteirinha, MG E-mail: ebsilva@nortecnet.com.br

3 Enga Agra, MSc., Pesquisadora EPAMIG/CTNM, C.P. 12, 39440-000, Nova Porteirinha, MG 
de Solos e Folha da EPAMIG/CTNM, Nova Porteirinha-MG, trazidas por produtores. Estas amostras eram procedentes de bananais da Região do Norte de Minas Gerais, com predomínio da cultivar Prata-Anã.

A amostragem foliar foi tomada segundo o método MEIR, citado por Silva et al. (1999), o qual usa a terceira folha verdadeira a contar do ápice de plantas que estejam com a inflorescência no estádio intermediário entre sua emissão e no máximo com todas as pencas femininas descobertas. Desta folha retiram-se de 10 a $15 \mathrm{~cm}$ da parte interna mediana do limbo, eliminando-se a nervura central

Os teores dos nutrientes nas folhas foram assim determinados: os teores de nitrogênio total das amostras foram determinados pelo método micro Kjeldahl, segundo metodologia descrita por Malavolta et al. (1997). No extrato, obtido por digestão nitroperclórica, descrita por Malavolta et al. (1997), foram dosados os teores totais de $\mathrm{P}$, por colorimetria, conforme Braga \& Defelipo (1974); os teores de Ca, Mg, Cu, Fe, Mn e Zn, por espectrofotometria de absorção atômica; os de K, por fotometria de chama; os de $\mathrm{S}$ total, por turbidimetria (Blanchar et al., 1965). O B foi extraído por incineração e determinado por colometria de curmumina, descrita por Malavolta et al. (1997).

As análises estatísticas constaram da avaliação do teor médio, mínimo e máximo, com intervalo de confiança a $5 \%$ para média e coeficiente de variação dos dados dos teores de N, P, K, $\mathrm{Ca}, \mathrm{Mg}, \mathrm{S}, \mathrm{B}, \mathrm{Cu}, \mathrm{Fe}, \mathrm{Mn}, \mathrm{Zn}$ e Na das amostras foliares. Realizouse um estudo de freqüência de ocorrência de bananais com teor deficiente, adequado e excessivo, tomando-se por base os teores para a cultura da bananeira propostos por Prezotti (1992), conforme Tabela 1.

Os dados de composição foliar da bananeira, segundo sua dispersão, intervalos de confiança $(\mathrm{P}<0,05)$, coeficiente de variação (CV em \%) e médias dos dados encontrados para cada nutriente e sódio são mostrados na Tabela 2.

Dentre os macronutrientes, o teor de Ca nas amostras avaliadas foi o que mais variou, tendo apresentado um extremo de teor máximo de 1,7 vez maior que o limite máximo da faixa proposta por Prezotti (1992), conforme mostrado na Tabela 1. Uma possível explicação é que isto se deva à utilização de água de poço com elevada quantidade de Ca na irrigação de algumas áreas e também à época de coleta feita pelos produtores entre janeiro e dezembro de 1999, com efeito das condições climáticas.

Dentre os micronutrientes, o $\mathrm{Cu}$ e $\mathrm{Mn}$ foram os que mais variaram, tendo sido observados teores 2,8 e 1,6 vezes maior que o limite máximo da faixa mostrada na Tabela 1 , respectivamente. Possivelmente, esta variação esteja ocorrendo pela pulverização de defensivos à base de $\mathrm{Cu}$ no controle fitossanitário, proporcionando um resíduo nas folhas analisadas, dificilmente removido pela lavagem das folhas; no caso do $\mathrm{Mn}$, pela efeito da umidade maior no período chuvoso, diferenças no manejo da irrigação e tipo de solo.

O sódio (Na) apresentou uma elevada variação (92,5\%) dos teores avaliados nas folhas da bananeira, sendo que o mínimo foi de $10 \mathrm{mg} \mathrm{kg}^{-1}$ e o máximo, de $1348 \mathrm{mg} \mathrm{kg}^{-1}$. O teor máximo é 8 vezes maior que o teor crítico proposto por Moreira (1999), de $165 \mathrm{mg} \mathrm{kg}^{-1}$.

Na Figura 1, é mostrada a freqüência de bananais com teor deficiente, adequado e excessivo para cada nutriente. Verificase que os bananais estão deficientes em ordem decrescente, para os macronutrientes $\mathrm{K}=\mathrm{S}>\mathrm{P}>\mathrm{N}>\mathrm{Mg}>\mathrm{Ca}$ e para os micronutrientes $\mathrm{Zn}>\mathrm{Cu}>\mathrm{Fe}>\mathrm{Mn}>\mathrm{B}$, ao contrário do encontrado por Malburg et al. (1984), em que os níveis foliares estiveram dentro da faixa de normalidade. Pode-se destacar o Ca, que está adequado em $98 \%$ das amostras foliares. Essa tendência é facilitada pela extensa faixa proposta como adequada por Prezotti (1992), que é de 2,5 a 12,0 $\mathrm{g} \mathrm{kg}^{-1}$, enquadrando a maioria das amostras.

Entre os micronutrientes observados com teor adequado, o Mn apresenta-se em maior porcentagem (87\%) dos bananais analisados. Entre os macronutrientes, isto foi verificado para o Ca foliar (98\%), ao contrário do encontrado por Gallo et al. (1974) para Nanica e Nanicão, de quase $100 \%$ de deficiência. O B apresentou-se em excesso nos bananais levantados, aparecendo um teor máximo de $55,9 \mathrm{mg} / \mathrm{kg}$ (Tabela 2), o que provavelmente seja uma adubação excessiva desse micronutriente efetuada pelos produtores da Região.

Além do exposto, para cada nutriente em particular, esses dados refletem de certo modo as propriedades químicas do solo e servem para a caracterização de sua fertilidade, pois, das amostras foliares observadas, $36 \%$ apresentaram algum tipo de deficiência de macronutrientes e 95\% apresentaram alguma deficiência de micronutriente (Figura 2). De modo geral, 97\% das amostras foliares apresentaram algum tipo de deficiência, mostrando a necessidade de melhorar o manejo nutricional dos bananais (Figura 2).

Estes valores mostraram que as faixas críticas para as condições da bananeira irrigada do Norte de Minas Gerais devem sofrer alguns ajustes e/ou então haver melhoraria no manejo nutricional dos bananais por parte dos produtores, sendo que este levantamento é somente um diagnóstico da situação da Região.

Pode-se concluir, portanto, que existe uma grande variação nos teores foliares de nutrientes, nos bananais irrigados do Norte de Minas Gerais. A grande maioria dos bananais apresentam

TABELA 1 - Faixas padrões de macro e micronutrientes da terceira folha da bananeira

\begin{tabular}{llll}
\hline Macronutriente & Faixa adequada $(\mathrm{g} / \mathrm{kg})$ & M icronutriente & Faixa adequada $(\mathrm{mg} / \mathrm{kg})$ \\
\hline $\mathrm{N}$ & $27-36$ & $\mathrm{~B}$ & $10-25$ \\
$\mathrm{P}$ & $1,8-2,7$ & $\mathrm{Cl}$ & - \\
$\mathrm{K}$ & $30-27$ & $\mathrm{Cu}$ & $6-30$ \\
$\mathrm{Ca}$ & $2,5-12$ & $\mathrm{Fe}$ & $80-360$ \\
$\mathrm{Mg}$ & $3-6$ & $\mathrm{M} \mathrm{n}$ & $200-2000$ \\
$\mathrm{~S}$ & $2-3$ & $\mathrm{Zn}$ & $20-50$ \\
\hline
\end{tabular}

Fonte: Prezotti (1992) 
TABELA 2 - Valores mínimo e máximo, média e intervalo de confiança dos teores foliares na terceira folha da bananeira de 1099 amostras. EPAMIG, Nova Porteirinha-MG, 2000.

\begin{tabular}{|c|c|c|c|c|c|}
\hline Teor & Mín imo & M áximo & M édia & $\begin{array}{c}\text { Intervalo de con fiança } \\
(P<0,05)\end{array}$ & $\mathrm{CV}(\%)^{1}$ \\
\hline $\mathrm{N}$ & 10 & 40 & 26 & $25-26$ & 16,8 \\
\hline $\mathrm{P}$ & 0,7 & 3,1 & 1,7 & $1,72-1,68$ & 15,0 \\
\hline $\mathrm{K}$ & 4 & 50 & 28 & $27-28$ & 21,4 \\
\hline $\mathrm{Ca}$ & 1,9 & 20,1 & 6,0 & $5,9-6,1$ & 33,5 \\
\hline $\mathrm{Mg}$ & 0,86 & 7,25 & 3,39 & $3,35-3,44$ & 22,1 \\
\hline S & 0,56 & 3,51 & 1,87 & $1,85-1,89$ & 19,5 \\
\hline B & 3,1 & 55,9 & 33,9 & $33,5-34,2$ & 16,7 \\
\hline $\mathrm{Cu}$ & 0,4 & 84,7 & 7,6 & $7,2-8,0$ & 91,3 \\
\hline $\mathrm{Fe}$ & 19,4 & 961,0 & 102,4 & $98,5-106,4$ & 65,4 \\
\hline $\mathrm{M} \mathrm{n}$ & 24 & 3186,0 & 540,3 & $514,3-566,3$ & 81,3 \\
\hline $\mathrm{Zn}$ & 7,4 & 71,5 & 19,2 & $18,7-19,7$ & 44,9 \\
\hline $\mathrm{N} \mathrm{a}$ & 10 & 1348,0 & 272,6 & $257,7-287,5$ & 92,5 \\
\hline
\end{tabular}

${ }^{1} \mathrm{CV}-$ Coeficiente de variação

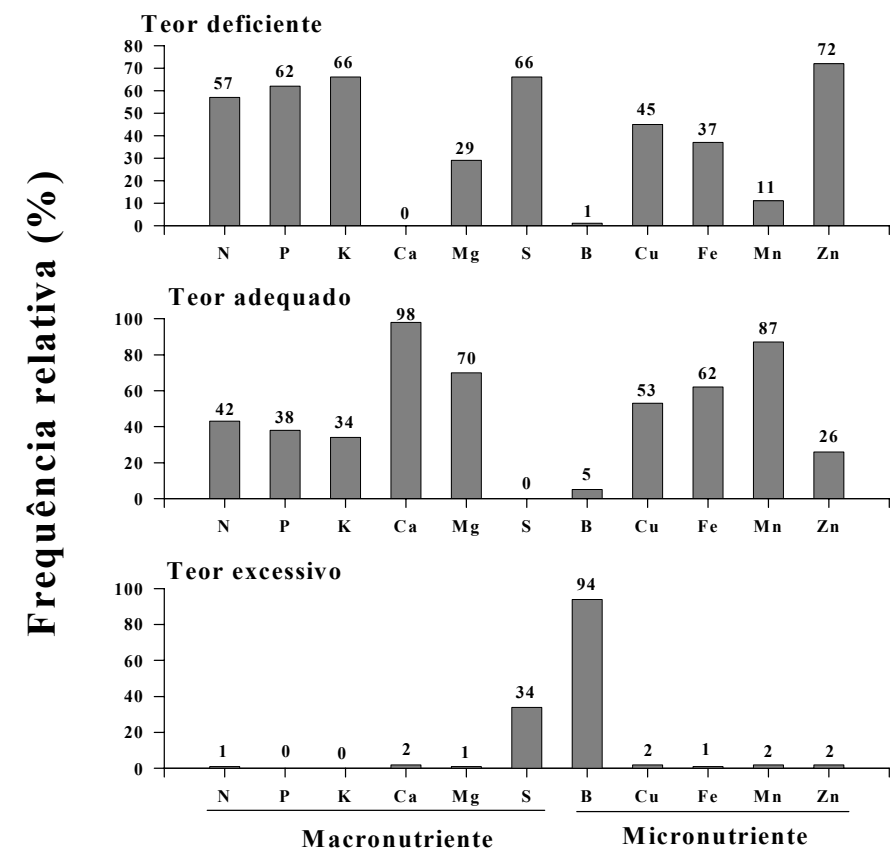

FIGURA 1 - Distribuição de freqüência de bananais com teor de nutrientes deficiente, adequado e excessivo na terceira folha da bananeira. EPAMIG, Nova Porteirinha-MG, 2000.

deficiências minerais. A deficiência em micronutrientes é mais freqüente do que a de macronutrientes. O B apresentou-se com teores excessivos e o $\mathrm{Zn}$ com teores deficientes na maioria das amostras foliares. O teor foliar de $\mathrm{Na}$ variou de $10 \mathrm{a} 1348 \mathrm{mg} \mathrm{kg}^{-1}$.

\section{REFERÊNCIAS BIBLIOGRÁFICAS}

BLANCHAR, R.W.; REHM, G.; CALDWELL, A.C. Sulfur in plant material digestion with nitric and perchloric acids. Soil Science Society of America Proceedings, Madison, v.29, n.1, p.71-72, 1965.

BORGES, A.L.; SILVA, S. O. Extração de macronutrientes por

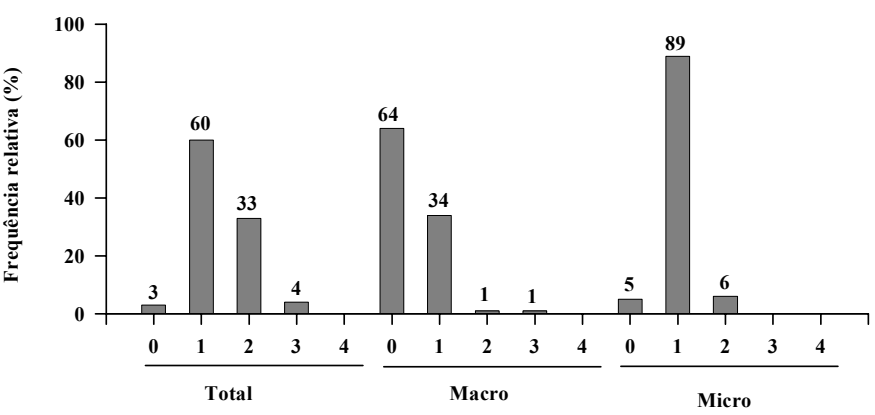

FIGURA 2 - Distribuição de freqüência de bananais com diferentes números de nutrientes deficientes para total, macro e micronutrientes na terceira folha da bananeira. EPAMIG, Nova Porteirinha-MG, 2000.

cultivares de banana. Revista Brasileira de Fruticultura, Cruz das Almas, v.17, n.1, p.57-66, 1995.

BRAGA, J.M.; DEFELIPO, B.V. Determinação espectrofotométrica de fósforo em extratos de solos de plantas. Revista Ceres, v. 21, p.73-85, 1974.

GALLO, J.R.; BATAGLIA, O.C.; FURLANI, P.R.; HIROCE, R.; FURLANI, A.M.C.; RAMOS, M.T.B., MOREIRA, R.S. Composição química inorgânica da bananeira (Musa acuminata Simmonds, cultivar Nanicão). Ciência e Cultura, São Paulo, v.24, n.1, p.70-79, 1972.

GALLO, J.R.; HIROCE, R.; BATAGLIA, O.C.; FURLANI, P.R.; FURLANI, A.M.C.; RAMOS, M.T.B., MOREIRA, R.S. Situação nutricional de bananais do Estado de São Paulo. Ciência e Cultura, São Paulo, v.26, n.4, p.355-259, 1974.

MALAVOLTA, E. ABC da análise de solos e folhas. São Paulo: Ceres, 1992. 124p.

MALAVOLTA, E.; VITTI, G.C.; OLIVEIRA, S.A. Avaliação do estado nutricional das plantas: princípios e aplicações. Piracicaba: POTAFOS, 1997. 201p. 
MALBURG, J.L.; HICHTEMBERG, L.A.; ANJOS, J.T.; UBERTI, A.A.A. Levantamento do estado nutricional de bananais catarinenses. In: CONGRESSO BRASILEIRO DE FRUTICULTURA, 7, 1983, Florianópolis, SC. Anais... Florianópolis, SC: SBF/EMPASC, 1984. v.1.p. 256-276.

MARTIN-PRÉVEL, P. Les éléments minéraux dans les bananier et dans son régime. Fruits, Paris, v.17, n.3, p. 123-128, 1962.

MARTIN-PRÉVEL, P., LACOEUILHE, J.; MARCHAL, J. Les éléments minéraux dans les bananier 'Gros Michel' au Cameroun. Fruits, Paris, v.23, n.5, p. 123-128, 1968.
MONTAGUT, G.; MARTIN-PRÉVEL, P. Besoin en engrais des bananeraies antillaises. Fruits, Paris, v.20, n.6, p. 265-273, 1965.

MOREIRA, R. S. Banana: teoria e prática de cultivo. 2. Ed. São Paulo: Fundação Cargill, 1999. (CDROOM)

PREZOTTI, C. Recomendações de calagem e adubação para o Estado do Espírito Santo. $3^{0}$ aproximação. Vitória: EMCAPA, 1992. 73p. (Circular Técnica, 12).

SILVA, J.T.A. da; BORGES, A.L.; MALBURG, J.L. Solos, Adubação e Nutrição da Bananeira. Informe Agropecuário, Belo Horizonte, v.20, n.196, p21-36, jan/fev. 1999. 